\title{
A Simple Approach to Assess if a Financial "Bubble" is Present: The Case of Bitcoin
}

\author{
Vitor M. A. da Fonseca ${ }^{1}$ \& Manuel A. R. da Fonseca ${ }^{2}$ \\ ${ }^{1}$ MBA Program in Finance and Risk Management, Federal University of Rio de Janeiro (UFRJ) \\ ${ }^{2}$ Faculty of Business Administration and Accounting, Federal University of Rio de Janeiro \\ Correspondence: Manuel A. R. da Fonseca, Faculty of Business Administration and Accounting, Federal University of \\ Rio de Janeiro.
}

$\begin{array}{lrr}\text { Received: April 4, } 2019 & \text { Accepted: April 30, } 2019 & \text { Available online: May 14, } 2019 \\ \text { doi:10.11114/aef.v6i4.4266 } & \text { URL: https://doi.org/10.11114/aef.v6i4.4266 }\end{array}$

\begin{abstract}
This article's goal is to evaluate if the recent price behavior of Bitcoin can be characterized as a financial market "bubble". To deal with this assessment, we adopt a statistical definition of a "bubble" derived from the efficient market hypothesis and we propose a simple method to test this proposition, based on the time-series model known as random walk. We analyze the data available for Bitcoin prices, together with an asset selected as benchmark, and perform statistical tests derived from simple regression equations. The main conclusion is that there is consistent evidence that that Bitcoin follows the pattern of a financial "bubble" - at least, such pattern is more evident in the case of Bitcoin than in the stock index used as benchmark.
\end{abstract}

Keywords: Bitcoin, "bubbles" in financial markets, time series models, statistical regression analysis

\section{Introduction}

What is Bitcoin? This question has recently gained increasing interest with the astonishing gains in value of this "created-by-technology" financial asset. In fact, several answers can be offered: It is a secretive (crypto) currency, a new and revolutionary commodity, an original and strikingly different "model of trust", a currency resulting from a decentralized network of qualified participants, a convenient form of payment based on new digital technologies, and so on.

Despite the diverse explanations for the Bitcoin phenomenon, many analysts appear to share the view that, whatever this new asset really is, it is most likely just another case of a financial "bubble" - and, of course, one that may burst at any moment. ${ }^{1}$ The main goal of this paper is, then, to evaluate this proposition - i.e. that the recent price behavior of Bitcoin can be characterized as a "bubble" in a financial market.

In order to deal with this assessment, we adopt a technical definition of "bubble" derived from the efficient market hypothesis (Section 2), and we propose a simple method to test this proposition, based on the time-series model best known as random walk (Section 3). Next, we analyze the data available for Bitcoin prices, together with an asset selected as benchmark, and perform statistical tests derived from simple regression equations (Section 4). Finally, we make an additional comparison with one of the clearest standards of "bubbles" - the trajectory of the Nasdaq Composite Index in the 1990s.

The results obtained indicate that there is evidence that Bitcoin is indeed a recent case of a financial "bubble" - at least, there is evidence that it is more so than a benchmark stock index for small-capitalization companies.

\subsection{Review of the Literature}

Baur, Hong and Lee (2017) evaluate if Bitcoin is more properly a currency or an investment medium and they obtain correlations among 17 international financial assets. Based on their results, they conclude that Bitcoins are mainly used as a form of investment, and not as a medium of exchange. Glaser, Zimmermann, Haferkorn, Weber and Siering (2014) question the uses of bitcoin for other purposes besides financial investment. The authors claim to have found strong

1 “[... As bitcoin's value appreciates [... it] begins to look like a speculative bubble." (White, Marinakis and Walsh, 2018, p. 2). 
evidence that users do not view bitcoin as a transaction facilitator but mainly as a speculative investment opportunity.

Urquhart (2016), in turn, investigates the market efficiency of Bitcoin - the general objective is, therefore, similar to the one pursued in this article - and he develops different types of statistical testes based on return rates, but his results are not conclusive. In a different perspective, Garcia, Tessone, Mavrodiev and Perony (2014) also deal with the possibility of Bitcoin's price record being a form of a financial "bubble". Their analysis includes a vector auto-regression (VAR) applied to four types of variables - Bitcoin data, social media activity, search volumes in Google and user adoption of Bitcoin - and they identify two reinforcing loops, or cycles, in this set of variables.

On the other hand, Brandvolt, Molnár, Vagstad and Valstad (2015) investigate the relative role of the main Bitcoin exchanges in terms of influencing market price. Bank for International Settlements (2018) mentions pitfalls and risks to cryptocurrencies and its holders. It affirms that the required trust on a cryptocurrency - that is by its nature unredeemable - can quickly dissipate because of the decentralized aspect of it. Another complication of decentralization is the lack a central issuer working to minimize volatility.

White, Marinakis, and Walsh (2018) select arguably acceptable cases of bubbles to compare with the case of bitcoin. The authors show that bitcoin exceeds in price appreciation all the selected cases, cautioning that, from a historical perspective, the rapid appreciation of bitcoin has shown to be unsustainable. Gervais, Karame, S. Capkun and V. Capkun (2014) elaborate that the decentralization - which at first is one of the selling points of Bitcoin - brings unknown risks. The authors found that more than $75 \%$ of Bitcoin mining power is controlled by only 6 pools. This goes against the original idea that decentralized operation and transparency would generate security and trust. In relation to problems not directly linked to decentralization, Moore and Christin (2012) examine 40 Bitcoin exchanges and find that 18 had been closed, with customer account balances often lost. From a different perspective, Brezo and Bringas (2012) list manners in which Bitcoin can be used in unlawful situations, pointing out that it lends itself to money laundering activities.

\section{Definitions of Financial "Bubble"}

Although the idea that the market behavior of an asset may be considered a "bubble" is a common place in academic research as well as in the popular media, apparently there is not a generally accepted definition of a financial "bubble". It is quite common to find technical articles discussing price behaviors that can be characterized as "bubbles", together with several historical experiences, and nowhere a clear characterization of this type of phenomenon is provided. There are, nevertheless, exceptions to this rule and Contessi and Kerdnunvong (2015) explore different definitions that have been suggested - although, from a scientific perspective, they can very well be questioned. ${ }^{2}$

Further, in a technical letter provided by the Chicago Fed, the following definition is advanced:

"What are asset bubbles? In general, [...] a bubble exists when the market price of an asset exceeds its price determined by fundamental factors by a significant amount for a prolonged period." ${ }^{3}$

Clearly, in this case, the burden of defining a "bubble" is transferred to the effort of specifying what exactly is a "price determined by fundamental factors". In any case, the authors also suggest a comparison with asset prices that behave according to the efficient market hypothesis.

"The efficient market hypothesis asserts that [...] actual and fundamental prices are always the same, and bubbles cannot exist unless they are driven by irrational behavior or market rigidities [...]"4

That is, based on this argument, "bubbles" occur when prices do not behave according to the efficient market hypothesis, which establishes that asset prices - and, in particular, stock prices - change only in response to new and unpredictable information. Therefore, these price changes must also behave in an unpredictable fashion. More specifically, the essence of the proposition known as the "efficient market hypothesis" is that asset prices should follow a random walk - i.e. that price changes should be random and unpredictable. ${ }^{5}$

\section{Random Walk and Statistical Tests: A Methodological Proposition}

A time-series model in which the value of a variable in one period is equal to its value in the previous period plus a

\footnotetext{
2 For example, in one case, a "bubble" is defined as "an upward price movement over an extended range that then implodes." Thus, according to this definition, one could only ascertain the existence of a "bubble" after the fact - that is, after it burst.

${ }^{3}$ Evanoff, Kaufman, and Malliaris (2012), p. 1.

4 Ibid.

${ }^{5}$ Bodie, Kane and Marcus (2009).
} 
random error is called a random walk. Such a model can be represented by the following equation: ${ }^{6}$

$$
x_{\mathrm{t}}=x_{\mathrm{t}-1}+e_{\mathrm{t}}
$$

The assumptions imposed on the random variables $e_{\mathrm{t}}$ are:

a. Zero mean: $\mathrm{E}\left(e_{\mathrm{t}}\right)=0$;

b. Constant variance: $\mathrm{E}\left(e_{\mathrm{t}}^{2}\right)=\sigma^{2}$;

c. Uncorrelated errors: $\mathrm{E}\left(e_{\mathrm{t}} e_{\mathrm{s}}\right)=0$, if $\mathrm{t} \neq \mathrm{s}$.

It is not difficult to show that, as a consequence of these assumptions, the following result applies to the variance of $x_{\mathrm{t}}$ :

$$
\operatorname{Var}\left(x_{\mathrm{t}}\right)=(\mathrm{t}-1) \sigma^{2}
$$

That is, as $t \rightarrow \infty$, this variance becomes infinitely large. To deal with this problem, a simple transformation is commonly used:

$$
y_{\mathrm{t}}=x_{\mathrm{t}}-x_{\mathrm{t}-1}=e_{\mathrm{t}}
$$

Therefore, one approach that can be used to assess if the price behavior of an asset should be described as a "bubble" is to evaluate if the first differences of the daily prices follow the assumptions used for the random errors $e_{\mathrm{t}}$ and, in special, if these variables have a constant variance (assumption b), and if variables in different (successive) periods are uncorrelated (assumption c).

Given the standard definitions of the population correlation coefficient, $\rho$, and its sample estimator, $r$, a statistical test is available to assess the hypothesis $\mathrm{H}_{0}: \rho=0 .^{7}$ However, in a more convenient approach, based on the relation between correlation and regression analyses, a simple regression equation can be used. ${ }^{8}$ Considering that the assumptions of regression analysis are valid - in the very common case that the regressor is also a random variable -, the following result can be demonstrated for the regression equation $Z=\alpha+\beta W+\varepsilon$ :

$$
\operatorname{Cov}(W, Z)=\beta \operatorname{Var}(W)
$$

Therefore, to test if the correlation coefficient (and the covariance) between $W$ and $Z$ is zero, it is sufficient to test the hypothesis $\mathrm{H}_{0}: \beta=0$. In this case, the following regression equation is used:

\section{Empirical Analysis and Results}

$$
y_{\mathrm{t}}=\beta y_{\mathrm{t}-1}+\varepsilon_{\mathrm{t}}
$$

In this Section, the methodology outlined in Section 3 is applied to Bitcoin price data. However, in order to obtain more

\begin{tabular}{|c|c|c|c|c|c|c|}
\hline & $\begin{array}{l}\text { Stocks } \\
\text { Small } \\
\text { Cap }^{1}\end{array}$ & $\begin{array}{l}\text { Stocks } \\
\text { Large } \\
\text { Cap }^{2}\end{array}$ & $\begin{array}{l}\text { T-Bonds } \\
\text { Long Maturity }\end{array}$ & $\begin{array}{l}\text { T-Bonds } \\
\text { Medium } \\
\text { Maturity }^{3}\end{array}$ & T-Bills & $\begin{array}{l}\text { Inflation } \\
\text { CPI }\end{array}$ \\
\hline Sample average ${ }^{4}$ & 12.1 & 9.9 & 5.5 & 5.3 & 3.6 & 3.0 \\
\hline Standard deviation & 32.6 & 20.4 & 9.5 & 8.3 & 3.1 & 3.6 \\
\hline Minimum & -52.7 & -45.5 & -8.7 & -5.8 & -1.6 & -10.2 \\
\hline Maximum & 187.8 & 54.6 & 32.7 & 33.4 & 15.0 & 18.1 \\
\hline Number of periods & 85 & 85 & 85 & 85 & 85 & 85 \\
\hline
\end{tabular}
meaningful conclusions, the Bitcoin data sample is compared with a benchmark - that is, an asset that can be considered a standard in relation to which other assets could be compared. In Table 1, summary statistics are presented for some financial indicators in the US market.

Table 1. Financial indicators in the US - annual data, 1926-2010. Rates of return, \%.

Notes: ${ }^{1}$ Russell 2000. ${ }^{2}$ S\&P 500. ${ }^{3}$ Data until 2001. ${ }^{4}$ Geometric average.

Source: Indicators obtained by the authors from original data in Bodie, Kane \& Marcus (2009) and in https://fred.stlouisfed.org.

According to Table 1, the index for stocks of smaller companies (Russell 2000) has shown, over the years, the greatest volatility among the main financial indicators available for the US market, and it was chosen as the benchmark for the

\footnotetext{
6 Shumway and Stoffer (2006). This is a especial case of the first order autoregressive, or AR(1), process $x_{\mathrm{t}}=\delta+\theta x_{\mathrm{t}-1}$ $+e_{\mathrm{t}}$.

7 Newbold (1984).

8 This approach is suggested in T. Wonnacott and R. Wonnacott (1972). It was used in Fonseca (2013).
} 
analysis of Bitcoin price data - which is also a very risky financial asset.

4.1 Russell 2000 - Statistical Analysis

In this analysis, data since 2009 was used - the start of the period when Bitcoin became available. From the original data, first differences were calculated (Figure 1). The main statistical indicators for this data-set appear below.

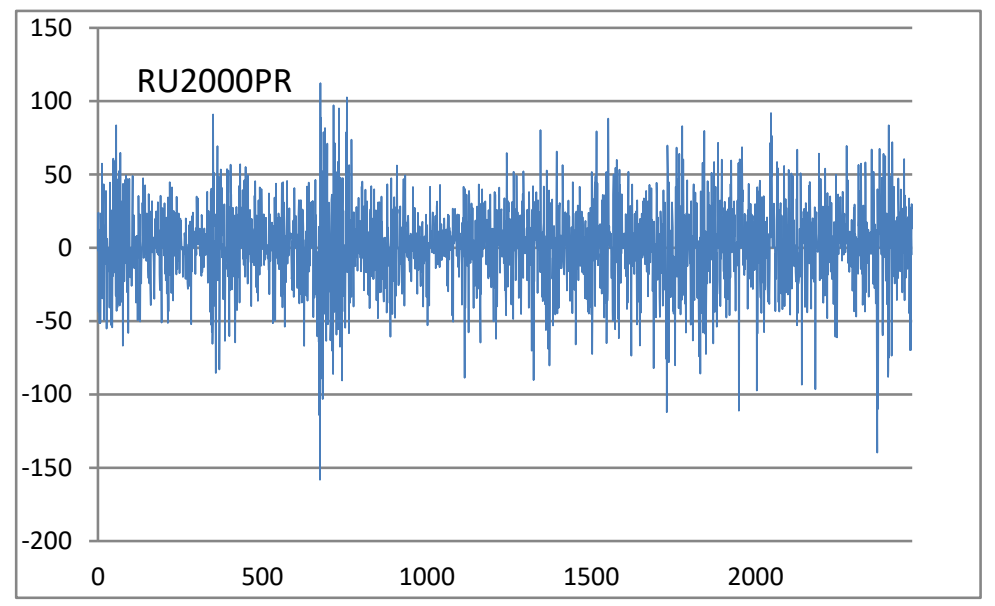

Figure 1. Russell 2000: First differences calculated from the original daily data, 2009-2018

Source: Calculated by the authors from original data in the Federal Reserve Bank of St. Louis. Available in https://fred.stlouisfed.org.

Table 2. Russell 2000 - Basic statistical indicators: First differences calculated from the original daily data, 2009-2018.

$\begin{array}{ll}1.305743 & \text { Average } \\ 0.604279 & \text { St. Error } \\ 2.7 & \text { Median } \\ 29.03686 & \text { St. Deviation } \\ 843.1391 & \text { Variance } \\ -0.34955 & \text { Asymmetry } \\ 1.455473 & \text { Kurtosis } \\ 270.54 & \text { Range } \\ -158.22 & \text { Minimum } \\ 112.32 & \text { Maximum } \\ 3014.96 & \text { Sum } \\ 2309 & \text { Count }\end{array}$

Source: Obtained by the authors.

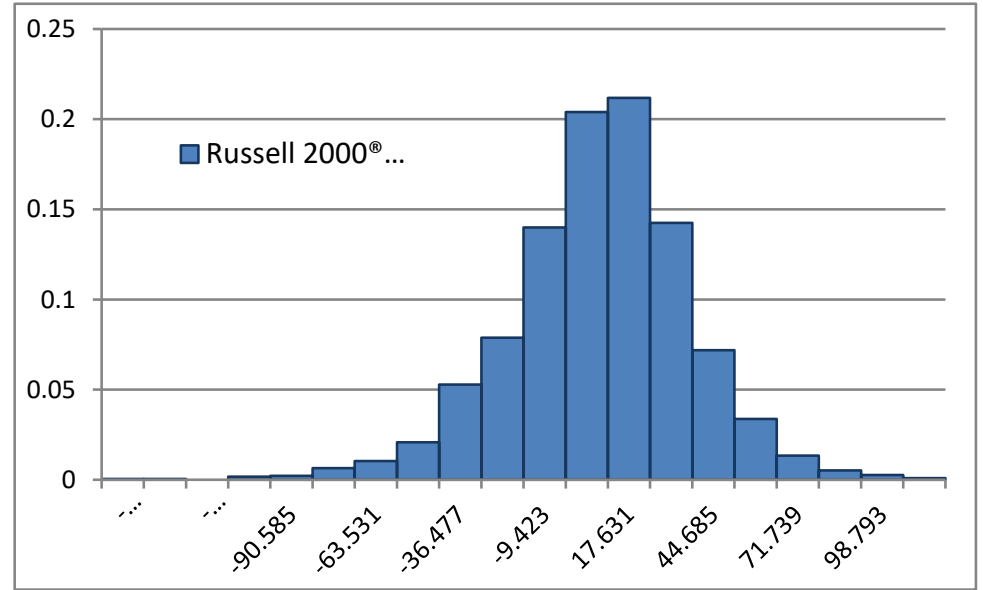

Figure 2. Russell 2000 - Histogram: First differences obtained from the original daily data, 2009-2018 Source: Obtained by the authors.

\subsection{Bitcoin - Statistical Analysis}

An initial examination of Bitcoin prices reveal a major structural change in the data set (Figure 3) - that is, considering 
the statistical properties, the latter period cannot be compared with the earlier one. Therefore, assumption (b) in the previous Section clearly is not valid. In order to mitigate this problem, the sample that was, in fact, used was initiated after the $1700^{\text {th }}$ observation. Figure 4 depicts the data used.

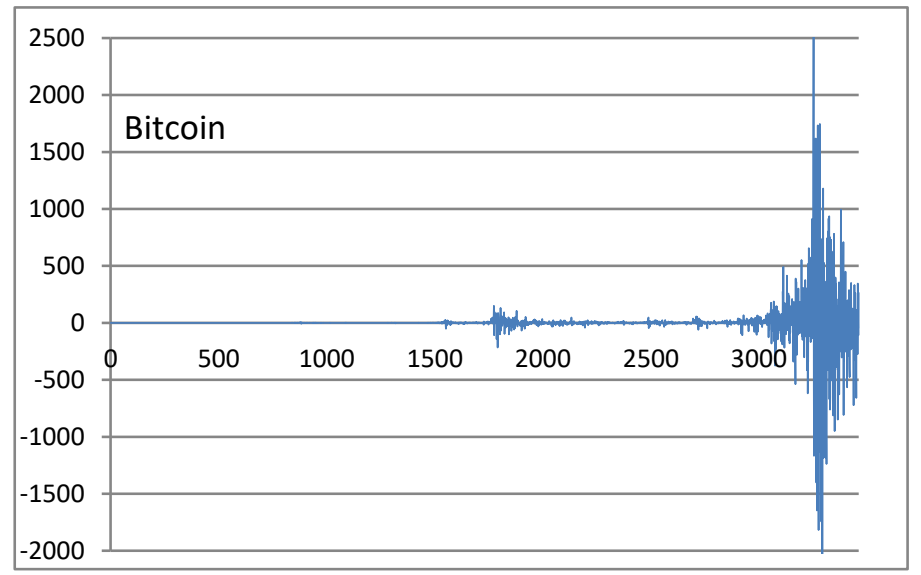

Figure 3. Bitcoin daily prices: First differences calculated from the original data, 2009-2018 Source: Calculated by the authors from original data available in https://charts.bitcoin.com/chart/price.

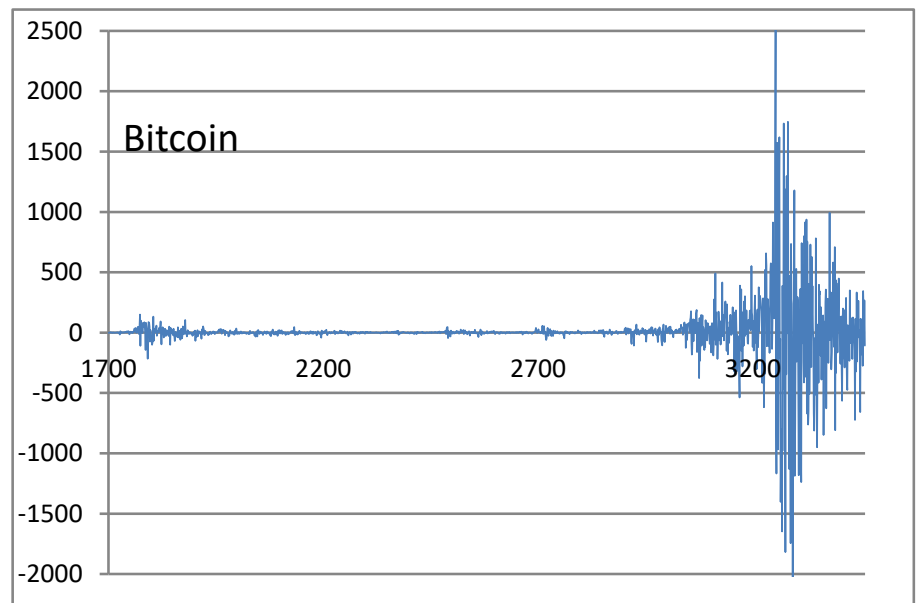

Figure 4. Bitcoin daily prices - Reduced sample: First differences calculated from the original data, 2013-2018 Source: Obtained by the authors.

As can be perceived from Figure 4, the sample available for Bitcoin prices, even including only the latter period, still presents major structural changes that, in statistical regression analysis, are described as heteroskedastic regression errors - i.e. assumption (b) fails. As it is well known (e.g. Verbeek, 2004), this problem in the errors of the regression equation poses serious problems for regression results and statistical tests, leading researchers to use alternatives for the standard regression methods.

Table 3. Bitcoin - Basic statistical indicators: First differences calculated from the original daily data, 2013-2018

\begin{tabular}{ll}
\hline 3.6115694 & Average \\
5.4410889 & St. Error \\
0.82 & Median \\
228.5904999 & St. Deviation \\
52253.6166 & Variance \\
0.52252559 & Asymmetry \\
36.0138685 & Kurtosis \\
4843.54 & Range \\
-2066.65 & Minimum \\
2776.89 & Maximum \\
6374.42 & Sum \\
1765 & Count \\
\hline
\end{tabular}

Source: Obtained by the authors. 


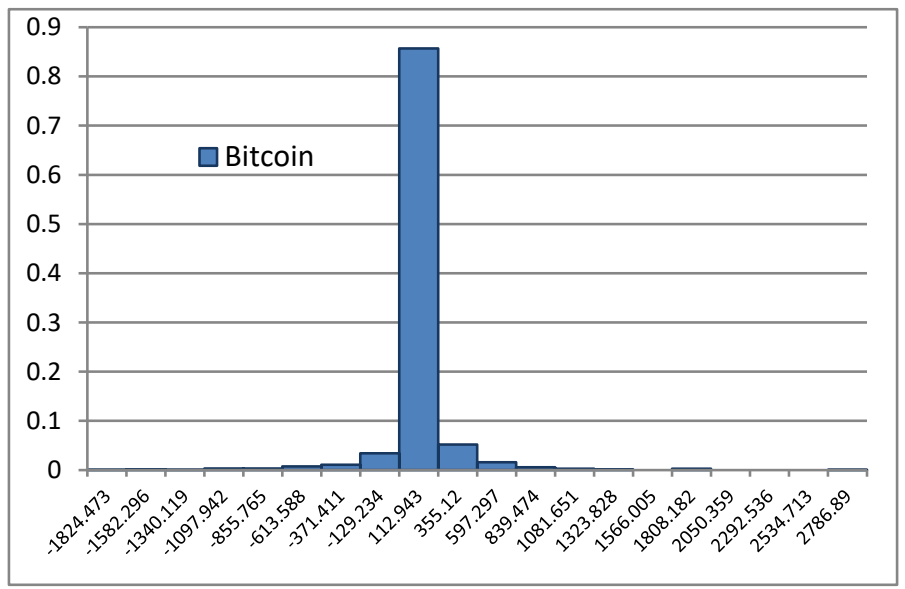

Figure 5. Bitcoin - Histogram: First differences obtained from the original daily data, 2013-2018

Source: Obtained by the authors.

\subsection{A Test of Hypothesis - Is the Price Behavior a "Bubble"?}

The main goal of this paper is to assess if the pattern of Bitcoin prices is in conformity with what most analysts may describe as a financial "bubble". To test for this possibility, as outlined in Section 3, we evaluate if the asset's price pattern behaves as predicted by the efficient market hypothesis - that is, if the statistical properties of the data series is in accordance with the random walk time-series model. More specifically, we test if the correlation between first differences in successive periods is zero. Further this test is set in the context of the statistical regression model. Therefore, in the regression equation (5) above, we test $\mathrm{H}_{0}: \beta=0$. The results obtained for Bitcoin prices, and for the Russell 2000 index, which is used as a benchmark, are shown below.

\subsubsection{Russel 2000}

The regression results for the benchmark are included in Table 4 and Figure 6.

Table 4. OLS, using observations 1-2476 $(\mathrm{T}=2223)$ Observations missing or incomplete were ignored: 253 Dependent variable: Russell

\begin{tabular}{|c|c|c|c|c|c|}
\hline & Coefficient & Standard Error & t-ratio & $\mathrm{p}$-value & \\
\hline Russell_1 & -0.0416852 & 0.0209984 & -1.985 & 0.0472 & ** \\
\hline Average dependent var. & \multicolumn{2}{|c|}{1.343315} & S.D. dependent var. & \multicolumn{2}{|c|}{28.94991} \\
\hline Sum squared residuals & \multicolumn{2}{|c|}{1862959} & S.E. of regression & \multicolumn{2}{|c|}{28.95541} \\
\hline R-square non-centered & \multicolumn{2}{|c|}{0.001770} & R- square centered & \multicolumn{2}{|c|}{-0.000380} \\
\hline $\mathrm{F}(1,2222)$ & \multicolumn{2}{|c|}{3.940877} & P-value(F) & \multicolumn{2}{|c|}{0.047249} \\
\hline
\end{tabular}

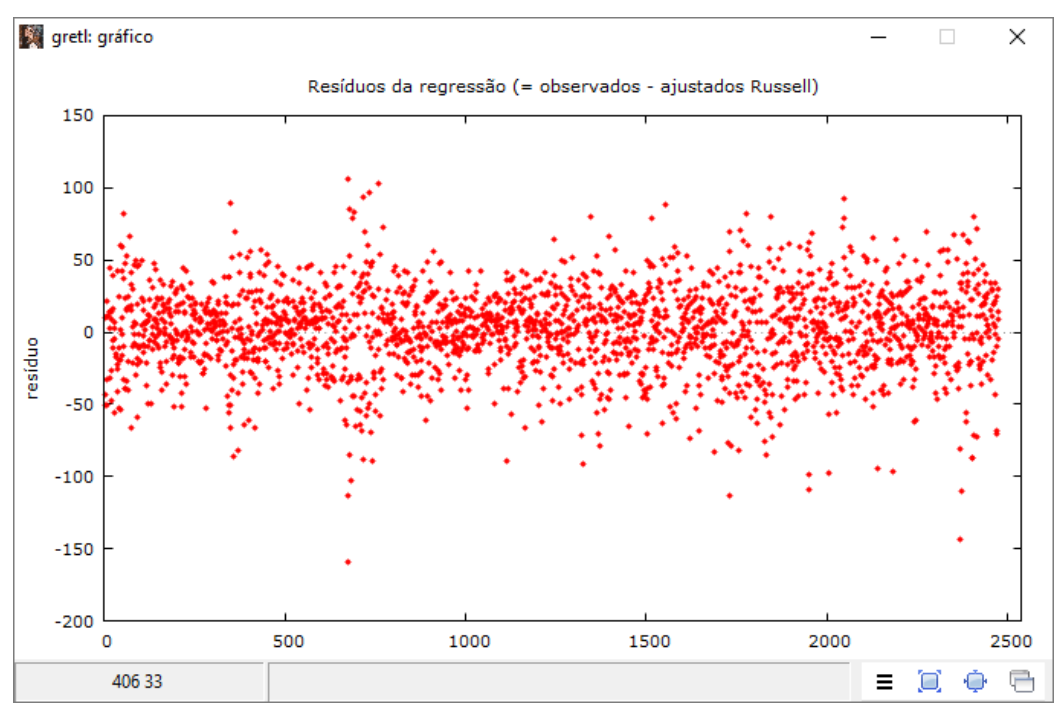

Figure 6. Residuals of regression in Table 4 
As we can see from Table 4, the hypothesis of zero correlation cannot be rejected only at the $1 \%$ significance level - but it can indeed be rejected at the 5\% significance level. Therefore, according to the analysis presented in this paper, one can accept with $95 \%$ confidence the alternative hypothesis that, since 2009, the Russell 2000 behaves as a financial "bubble".

\subsubsection{Bitcoin}

The regression results for Bitcoin prices are included in Table 5 and Figure 7.

Table 5. OLS, using observations 1700-3462 $(\mathrm{T}=1763)$ Dependent variable: Bitcoin

\begin{tabular}{lcccc}
\hline & Coefficient & Standard Error & t-ratio & p-value \\
\hline Bitcoin_1 & 0.0738327 & 0.0237595 & 3.108 & 0.0019 \\
Average dependent var. & 3.618712 & S.D. dependent var. & & 228.7201 \\
Sum squared residuals & 91695832 & S.E. of regression & 228.1245 \\
R-square non-centered & 0.005451 & R- square centered & 0.005201 \\
F(1, 1762) & 9.656590 & P-value(F) & 0.001917 \\
\hline
\end{tabular}

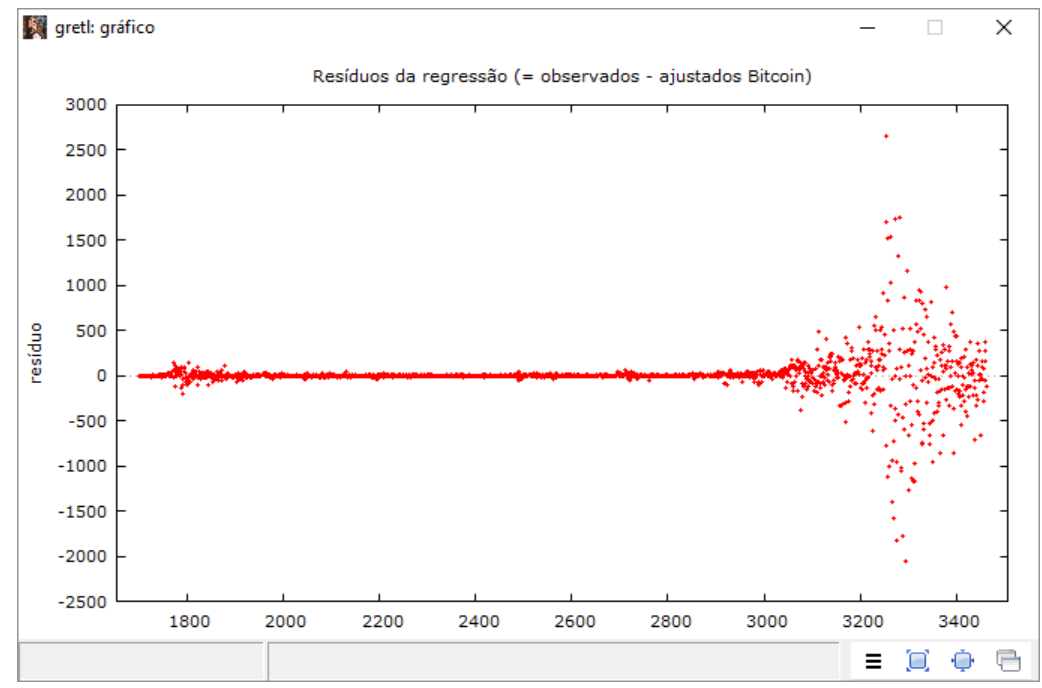

Figure 7. Residuals of regression in Table 5

As we can see from Table 5, the hypothesis of zero correlation can be rejected at virtually any significance level. Therefore, according to the analysis presented in this paper, one can accept with virtually $100 \%$ confidence the alternative hypothesis that, since 2013, Bitcoin behaves as a financial "bubble". In other words, although this latter result is affected by the presence of heteroskedastic errors, the available data suggest that there is considerably more evidence of a financial "bubble" in the case of Bitcoin than in the Russell 2000 index.

\section{A Further Comparative Analysis - The Case of Nasdaq}

Quite many people would probably agree that the label "mother of all bubbles" best fits the trajectory of the Nasdaq in the late 1990s - i.e., it represents one of the clearest cases of a financial "bubble" in recent times -, and that experience was named the "dot-com bubble". To bring an additional perspective to the analysis developed here, the regression analysis is also applied to Nasdaq Composite Index data. The results appear in Figures 8 and 9, and in Table 6. The pattern displayed in these Figures is strikingly similar to that of Bitcoin prices. As can be perceived from the Table, the results for Nasdaq are located in an intermediate position, between the Russell 2000 and the bitcoin results. 


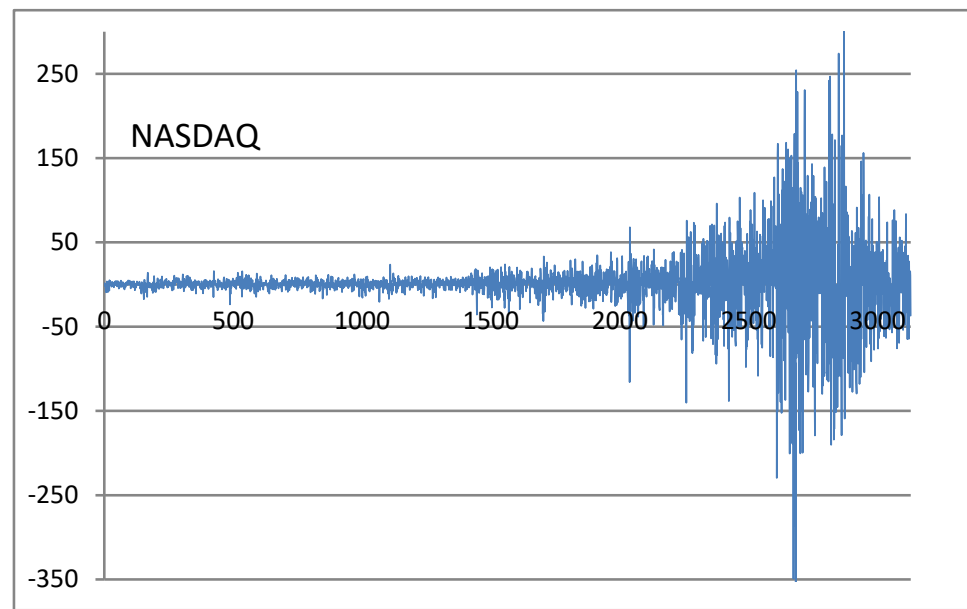

Figure 8. Nasdaq daily data: First differences calculated from the original data, 1990-2001 Source: Obtained by the authors.

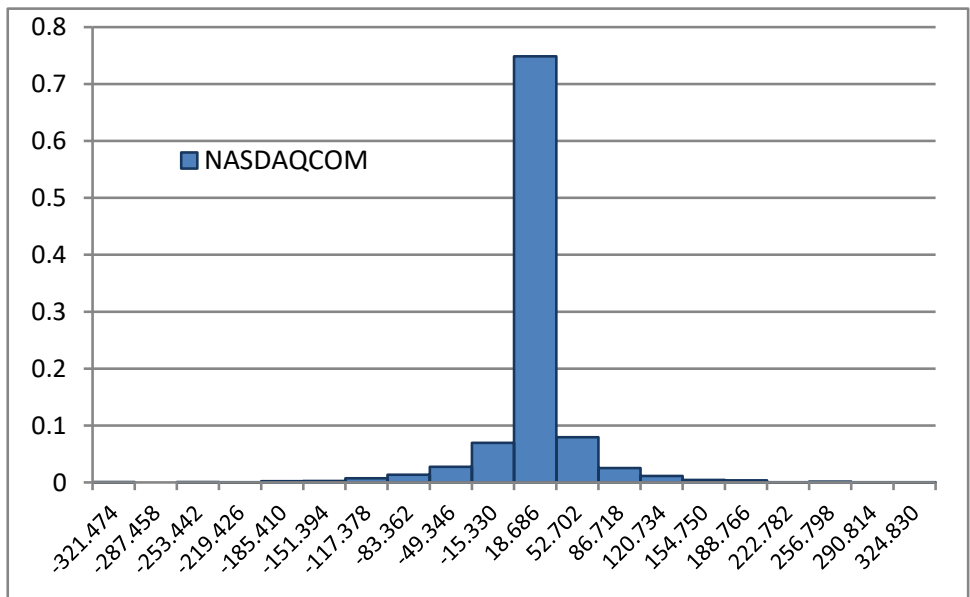

Figure 8. Nasdaq - Histogram: First differences obtained from the original daily data, 1990-2001 Source: Obtained by the authors.

Table 6. OLS, using observations 2-3129 $(\mathrm{T}=3128)$ Dependent variable: NASDAQCOM

\begin{tabular}{|c|c|c|c|c|c|}
\hline & Coefficient & Standard Error & t-ratio & $\mathrm{p}$-value & \\
\hline NASDAQCOM_1 & 0.0397683 & 0.0178713 & 2.225 & 0.0261 & ** \\
\hline Average dependent var. & \multicolumn{2}{|c|}{0.583792} & S.D. dependent var. & \multicolumn{2}{|c|}{38.19060} \\
\hline Sum squared residuals & \multicolumn{2}{|c|}{4554652} & S.E. of regression & \multicolumn{2}{|c|}{38.16486} \\
\hline R-square non-centered & \multicolumn{2}{|c|}{0.001581} & R- square centered & \multicolumn{2}{|c|}{0.001348} \\
\hline $\mathrm{F}(1,3127)$ & \multicolumn{2}{|c|}{4.951770} & P-value(F) & \multicolumn{2}{|c|}{0.026135} \\
\hline
\end{tabular}

\section{Conclusion}

In this paper, we propose a more precise, statistical definition to describe the situation when the price behavior of a financial asset should be characterized as a "bubble". According to a common view on the subject, a "bubble" exists in a financial market when the asset price exceeds its price determined by fundamental factors - by a significant amount and for a prolonged period. Moreover, the concept of a fundamental price is derived from the efficient market hypothesis - i.e., unless irrational behavior or market rigidities are present, the actual asset price coincides with the fundamental one.

From this basic proposition, we then use one of the main conclusions of the efficient market hypothesis to test if an asset price diverts or not from its fundamental price - i.e., to test if the dynamic path of the asset price follows the pattern of a random walk process. More specifically, we perform a statistical test to evaluate if the correlation coefficient for sequential first differences calculated from the asset price is zero - hypothesis of uncorrelated errors. The version of the test that was used is based on the analysis of simple regression equations.

In Section 4, the statistical test to evaluate zero correlation was applied to Bitcoin prices and to an asset used as a 
benchmark - the Russell 2000 small-capitalization stock index. We conclude that, in the case of Bitcoin, with close to $100 \%$ confidence, one can accept the alternative hypothesis of non-zero correlation - or, in other words, that since 2013 Bitcoin does not follow the dynamic pattern of a random walk process. However, in the case of the Russel 2000 index, we infer that, with no more than $95 \%$ confidence the alternative hypothesis of non-zero correlation should be accepted. Therefore, our main conclusion is that there is consistent evidence that Bitcoin follows the pattern of a financial "bubble" - at least, such pattern is more evident in the case of Bitcoin than in a benchmark stock index for small-capitalization companies. This conclusion is also supported by a comparative analysis of the trajectory of the Nasdaq Composite Index in the 1990s. There is very little controversy, if at all, with the assertion that, in the late 1990s, the dynamic path of that stock index was, in fact, consistent with a financial "bubble". Our results show that the statistical test applied to the Nasdaq index indicates it occupies an intermediate position in relation to Russel 2000 and Bitcoin.

This main conclusion is in agreement with White et al. (2018), who find that "[...] the bitcoin's rapid asset appreciation has exceeded the most prominently studied historical bubbles of the last three hundred years" (p. 19). The same basic conclusion, concerning autocorrelation, is reached in Baur et al. (2018): "[...] the Box-Pierce and Ljung-Box tests show highly significant test statistics at the $1 \%$ level indicating the presence of autocorrelation." (p. 182). However, in the case of Urquhart (2016), the results are not conclusive: "[...] evidence reveals that returns are significantly inefficient over our full sample, but when we split our sample into two subsample periods, we find that some tests indicate that Bitcoin is efficient in the latter period." (p. 80). Although Urquhart's general conclusions are not in agreement with our findings, in one aspect our results converge - that is, the more recent period of Bitcoin price data shows a pattern closer to a random walk than the overall sample data (Figure 4 and Table 5). More specifically, when we run the statistical test with Bitcoin's data from 2016 onwards - i.e. starting from the $2550^{\text {th }}$ observation -, the results are closer to those for the Nasdaq index full data sample (Figure 8 and Table 6).

\section{References}

Baur, D. G., Hong, K. H., \& Lee, A. D. (2018). Bitcoin: Medium of exchange or speculative assets? Journal of International Financial Markets, Institutions and Money, 54, 177-189.

https://doi.org/10.1016/j.intfin.2017.12.004

Bank for International Settlements (2018). Cryptocurrencies: looking beyond the hype. BIS Annual Report, Ch. 5. Available at https://www.bis.org/publ/arpdf/ar2018e5.htm.

Bodie, Z., Kane, A., \& Marcus, A. J. (2009). Investments ( $8^{\text {th }}$ ed). Boston: McGraw-Hill.

Brandvolt, M., Molnár P., Vagstad, K., \& Valstad, O. C. (2015). Price discovery on Bitcoin exchanges. Journal of International Financial Markets, Institutions and Money, 36, 18-35. https://doi.org/10.1016/j.intfin.2015.02.010

Brezo, F., \& Bringas, P. (2012). Issues and risks associated with cryptocurrencies such as Bitcoin. SOTICS 2012: The Second International Conference on Social Eco-Informatics. https://www.researchgate.net.

Contessi, S., \& Kerdnunvong, U. (2015). Asset bubbles: Detecting and measuring them are not easy tasks. Regional Economist - Fed of St. Louis.

Evanoff, D., Kaufman, G., \& Malliaris, A. (2012). Asset price bubbles: What are the causes, consequences, and public policy options? Chicago Fed Letter, 304.

Fonseca, V. da. (2013) Análise de Persistência em Fundos de Investimento no Brasil. Faculty of Economics, Universidade Federal Fluminense (UFF).

Garcia, D., Tessone, C. J., Mavrodiev, P., \& Perony, N. (2014). The digital traces of bubbles: feedback cycles between socio-economic signals in the Bitcoin economy. J. R. Soc. Interface, 11, 1-9. https://doi.org/10.1098/rsif.2014.0623

Gervais, A., Karame, G., Capkun, S., \& Capkun, V. (2014). Is bitcoin a decentralized currency? IEEE Security \& Privacy, 12(3), 54-60. https://doi.org/10.1109/MSP.2014.49

Glaser, F., Zimmermann, K., Haferkorn, M., Weber, M., \& Siering, M. (2014). Bitcoin - asset or currency? Revealing users' hidden intentions. Twenty Second European Conference on Information Systems, Tel Aviv. Available at https://ssrn.com/abstract=2425247.

Moore, T., \& Christin, N. (2013). Beware the middleman: Empirical analysis of Bitcoin-exchange risk. In: Sadeghi A. R. (ed.) Financial Cryptography and Data Security, Lecture Notes in Computer Science. Berlin: Springer. https://doi.org/10.1007/978-3-642-39884-1_3

Newbold, P. (1984). Statistics for Business and Economics. Englewood Cliffs-NJ, Prentice-Hall.

Shumway, R., \& Stoffer, D. (2006). Time Series Analysis and Its Applications, With R Examples (2 ${ }^{\text {nd }}$ ed.). New YorkNY: Springer. 
Urquhart, A. (2016). The inefficiency of Bitcoin. Economics Letters, 148, 80-82. https://doi.org/10.1016/j.econlet.2016.09.019

Verbeek, M. (2004). A Guide to Modern Econometrics ( $2^{\text {nd }}$ ed.). Chichester-West Sussex: John Wiley.

White, R., Marinakis, Y., \& Walsh, S. (2018). Is bitcoin a currency or a technology?. Discussion Paper no. 5, GPEF-UFRJ. Available in: http://modelosfinanceiros.com.br/publicacoes.

Wonnacott, T., \& Wonnacott, R. (1972). Introductory Statistics (2 $2^{\text {nd }}$ ed.). New York-NY: John Wiley.

\section{Copyrights}

Copyright for this article is retained by the author(s), with first publication rights granted to the journal.

This is an open-access article distributed under the terms and conditions of the Creative Commons Attribution license which permits unrestricted use, distribution, and reproduction in any medium, provided the original work is properly cited. 